\title{
NOTE ON THE DEGREE OF CONVERGENCE OF SEQUENCES OF ANALYTIC FUNCTIONS*
}

BY

\author{
J. L. WALSH
}

It is the object of this note first to present a general theorem (Theorem 1) on maxima and superior limits of sequences of analytic functions; this is essentially a result in the theory of subharmonic functions, presumably of some interest in itself. Second, Theorem 1 is employed to establish a sharper theorem (Theorem 2) on the precise degree of convergence of general sequences of analytic functions. Finally, these general results are used to establish a quite specific result (Theorem 3) on the degree of convergence of certain sequences of analytic functions in an annular or more general region. Parts of Theorem 3 have previously been established, but in a more restricted form and by another method. The present Theorem 3 is sufficiently general to dominate results established elsewhere by the author on maximal convergence of sequences of polynomials, $\uparrow$ on approximation by functions analytic in a given region, $\ddagger$ on interpolation by functions analytic in a given region, $\S$ and on maximal convergence of sequences of rational functions.|| Various other applications of Theorem 3 seem to suggest themselves and will presumably appear in the future. 1

The following treatment is chosen rather for elements of simplicity than for ultimate generality. Some extensions present themselves at once as obvious; others are scarcely less immediate. But even in its present form, Theorem

\footnotetext{
* Presented to the Society, December 30,1938, under the title Maximal convergence of sequences of rational functions; received by the editors October 2, 1939.

$\dagger$ Interpolation and Approximation by Rational Functions in the Complex Domain, American Mathematical Society Colloquium Publications, vol. 20, New York, 1935. All references in the present note not otherwise indicated are to this book.

$\ddagger$ Proceedings of the National Academy of Sciences, vol. 24 (1938), pp. 477-486.

$\S$ These Transactions, vol. 46 (1939), pp. 46-65.

\| These Transactions, vol. 47 (1940), pp. 254-292.

I In particular the writer hopes later to apply these methods to the study of such questions as overconvergence, gap theorems, and the analogues of Jentzsch's theorem.

No general study of these questions seems as yet to have been made for sequences $f_{n}(z)$ of the type considered in Theorem 3, as contrasted with the partial sums of a series $\sum a_{n} p_{n}(z)$ when the asymptotic character of the $p_{n}(z)$ is known. But certain results on this general study for the special case of maximal sequences of polynomials have recently been obtained by Dr. Z. I. Mosesson in his 1937 doctoral dissertation at Harvard University. Mosesson's results are still unpublished, but were obtained prior to those of the present note; for the special case of sequences of polynomials, they overlap slightly our Theorem 3. Mosesson employs a new form of the two-constant theorem.
} 
3 seems to be of sufficient power and importance to deserve a systematic and independent exposition.

The following theorem is largely an application of known principles; nevertheless the present form is highly convenient for reference:

Theorem 1. Let $R$ be a region whose boundary $B$ consists of a finite number of Jordan arcs $J_{k}$, mutually disjoint except possibly at end points $A_{j}$. Let the function $V(z)$ be harmonic and bounded in $R$, continuous in the corresponding closed region $\bar{R}$ except in the points $A_{j}$, constant on each $J_{k}$.

Let the functions $F_{n}(z), n=1,2, \cdots$, be uniformly bounded in $R$, locally analytic except perhaps for branch points although not necessarily single-valued in $R$, but let $\left|F_{n}(z)\right|$ be single-valued in $R$ and continuous in $\bar{R}$ except perhaps in the points $A_{j}$; and suppose we have for each $k$

$$
\underset{n \rightarrow \infty}{\limsup }\left[\text { l.u.b. }\left|F_{n}(z)\right|, z \text { on } J_{k}\right] \leqq\left[e^{V(z)}, z \text { on } J_{k}\right] \text {. }
$$

Then on every closed set $Q$ in $\bar{R}$ containing no point $A_{j}$ we have

$$
\limsup _{n \rightarrow \infty}\left[\max \left|F_{n}(z)\right|, z \text { on } Q\right] \leqq\left[\max e^{V(z)}, z \text { on } Q\right] \text {. }
$$

In the proof of Theorem 1 let us suppose for the moment that $V(z)$ and $\left|F_{n}(z)\right|$ are continuous in $\bar{R}$. If $\eta>0$ is arbitrary, we have from (1) for $n$ sufficiently large

$$
\log \left|F_{n}(z)\right| \leqq V(z)+\eta, \quad \quad z \text { on } B .
$$

To be sure, the function $F_{n}(z)$ may vanish in $\bar{R}$, so here and below the value $-\infty$ is contemplated as admissible in the first member of (3). The subharmonic character of the function $\log \left|F_{n}(z)\right|$ in $R$ now yields from (3)

$$
\log \left|F_{n}(z)\right| \leqq V(z)+\eta, \quad z \text { in } \bar{R},
$$

for $n$ sufficiently large, and (2) therefore follows.

If $V(z)$ and $\left|F_{n}(z)\right|$ are no longer assumed continuous in the points $A_{j}$, the reasoning just given is to be modified slightly. It is no loss of generality to assume, as we do, that $R$ is a finite region bounded by analytic Jordan curves; for this situation can be obtained by transforming the original configuration by a suitable conformal map. Let $\eta>0$ be arbitrary. Denote by $M$ a common upper bound for $\log \left|F_{n}(z)\right|$ and $V(z)$ in $R$, and denote by $W_{\nu}(z)$ a function which is harmonic in $R$, continuous in $\bar{R}$, equal to $2 M+\eta$ in each point $A_{j}$, never greater than $2 M+\eta$ on $B$, and equal to zero on $B$ except in the neighborhood of diameter $\nu$ of each point $A_{j}$. When the diameters $\nu$ of these neighborhoods approach zero, the function $W_{\nu}(z)$ approaches zero at every interior point of $R$, as follows from the representation of $W_{\nu}(z)$ in $R$ 
by Green's formula involving Green's function for $R$. For $n$ sufficiently large we have from (1)

$$
\text { [l.u.b. } \log \left|F_{n}(z)\right|, z \text { on } J_{k\rfloor} \leqq\left[V(z)+\eta, z \text { on } J_{k}\right] \text {. }
$$

The function $V_{n}^{\prime}(z) \equiv \log \left|F_{n}(z)\right|-V(z)-\eta$ is subharmonic in $R$. When $z$ in $R$ approaches $B$, the function $V_{n}^{\prime}(z)-W_{\nu}(z)$ has a superior limit not greater than zero, whether $z$ approaches a point $A_{j}$ or an interior point of $J_{k}$; it follows that the superior limit of this function in $R$ is not greater than zero:

$$
V_{n}^{\prime}(z) \leqq W_{\nu}(z), \quad z \text { in } R .
$$

This inequality is true for each $\nu$, so we have by allowing $\nu$ to approach zero,

$$
V_{n}^{\prime}(z) \leqq 0, \quad z \text { in } R,
$$

which is equivalent to the inequality (4), valid for $n$ sufficiently large at every point of $R$; by allowing $z$ in $R$ to approach $B$, we see that inequality (4) holds throughout $\bar{R}$, except in the points $A_{j}$ where $V(z)$ is not continuous. Inequality (2) follows, so Theorem 1 is completely established.

Theorem 1 persists if the functions $F_{n}(z)$ are not required to be continuous on the $\operatorname{arcs} J_{k}$ except in the points $A_{j}$, provided (1) is valid, where the square bracket in the first member of (1) is interpreted as the least upper bound of the set of limit values approached by $\left|F_{n}(z)\right|$ when $z$ in $R$ approaches an arbitrary point of $J_{k}$, end points of $J_{k}$ excepted. Similarly, if $Q$ contains points of $B$, the square bracket in the first member of (2) is to be interpreted as the least upper bound of the set of values approached by $\left|F_{n}(z)\right|$ as $z$ in $R$ approaches an arbitrary point of $Q$. The proof just given is valid in this more general case.

In a similar manner we may interpret and establish (2) even when the $F_{n}(z)$ are not required to be continuous on the $\operatorname{arcs} J_{k}$ except in the points $A_{j}$ and when $Q$ in $\bar{R}$ is allowed to contain points $A_{j}$.

A result complementary to Theorem 1 is now to be proved:

THEOREM 2. Under the conditions of Theorem 1 suppose the equality sign holds in (2) for a particular continuum $Q_{0}$ in $R$ ( $Q_{0}$ may be a single point):

$$
\underset{n \rightarrow \infty}{\limsup }\left[\max \left|F_{n}(z)\right|, z \text { on } Q_{0}\right]=\left[\max e^{V(z)}, z \text { on } Q_{0}\right] .
$$

Then on every continuum $Q$ in $\bar{R}$ consisting of more than one point and containing no point $A_{j}$ we have the corresponding equality:

$$
\limsup _{n \rightarrow \infty}\left[\max \left|F_{n}(z)\right|, z \text { on } Q\right]=\left[\max e^{V(z)}, z \text { on } Q\right] .
$$


Our proof of Theorem 2 is indirect. Let us suppose first that $Q$ lies in $R$. If (6) is not valid we may assume

$$
\begin{gathered}
\underset{n \rightarrow \infty}{\lim \sup }\left[\max \left|F_{n}(z)\right|, z \text { on } Q\right]=M, \\
M<\left[\max e^{V(z)}, z \text { on } Q\right] .
\end{gathered}
$$

We are to show the impossibility of (7) and (8). Replacing $Q$ by a proper subset of $Q$ (still a continuum not a single point) without altering the second member of (6) leaves us with a stronger statement to be proved and hence is allowable. Thus it is no loss of generality, since $V(z)$ is continuous in $\bar{R}$ except in the points $A_{j}$, to assume that we have

$$
V(z)>\log M,
$$

$z$ on $Q$.

We assume also, as we may do, that the complement of $Q$ is simply connected.

Denote by $V_{1}(z)$ the function harmonic and bounded in the region $R_{1}$ : $R-Q$, continuous and equal to $V(z)$ on each $J_{k}$ except in the points $A_{j}$, and continuous and equal to $\log M$ on the boundary points of $R_{1}$ which belong to $Q$. Theorem 1 in generalized form applies to the region $R_{1}$; for the conformal map $z=\Psi(w)$ of the complement of $Q$ onto the interior of a circle $C$ maps $R$ onto a region satisfying the original hypothesis of Theorem 1 . On the circle $C$ we have by (7)

$$
\limsup _{n \rightarrow \infty}\left[\max \left|F_{n}[\Psi(w)]\right|, w \text { on } C\right] \leqq M,
$$

provided the square bracket in the first member of (10) is interpreted as the least upper bound of all limit values of $\left|F_{n}[\Psi(w)]\right|$ as $w$ approaches $C$. For the region $R_{1}$ no point $A_{j}$ lies on $Q$. From Theorem 1 follows by (2) the inequality

$$
\underset{n \rightarrow \infty}{\limsup }\left[\max \left|F_{n}(z)\right|, z \text { on } Q_{0} \cdot \bar{R}_{1}\right] \leqq\left[\max e^{V_{1}(z)}, z \text { on } Q_{0} \cdot \bar{R}_{1}\right] ;
$$

a glance at the proof of Theorem 1 shows that the possible lack of one-to-oneness of the transformation $z=\Psi(w)$ on $Q$ and $C$ plays no role; the relation (4) valid on an open set in $R$ is valid also on the corresponding closed set. By virtue of (7) and (8) the corresponding inequality holds also in points of $Q_{0} \cdot Q$, provided we define $V_{1}(z)$ as equal to $\log M$ in the interior points of $Q$ :

$$
\underset{n \rightarrow \infty}{\lim \sup }\left[\max \left|F_{n}(z)\right|, z \text { on } Q_{0} \cdot Q\right] \leqq\left[\max e^{V_{1}(z)}, z \text { on } Q_{0} \cdot Q\right] \text {. }
$$

Combination of these two inequalities gives us

$$
\underset{n \rightarrow \infty}{\limsup }\left[\max \left|F_{n}(z)\right|, z \text { on } Q_{0}\right] \leqq\left[\max e^{V_{1}(z)}, z \text { on } Q_{0}\right]
$$


However, the function $V(z)-V_{1}(z)$ is harmonic and bounded in $R_{1}$, continuous in the corresponding closed region except in the points $A_{j}$, zero on the $J_{k}$, and by (9) is positive in the boundary points of $R_{1}$ which belong to $Q$. Consequently we have throughout $R_{1}$

$$
V(z)>V_{1}(z) \text {. }
$$

Inequality (12) is also valid in the points of $Q$, hence throughout $R$. The functions $V(z)$ and $V_{1}(z)$ are continuous on the closed set $Q_{0}$ in $R$, so we have

$$
\left[\max V(z), z \text { on } Q_{0}\right]>\left[\max V_{1}(z), z \text { on } Q_{0}\right] \text {. }
$$

Thus inequality (11) contradicts (5), and thereby equation (6) is established provided $Q$ lies in $R$.

The proof just given may require modification if $Q$ intersects $B$, as we now suppose. If $Q$ is identical with $\bar{R}$, the proof is immediate, for no point $A_{j}$ can exist and the function $V(z)$ is identically constant on each component of $B$; the negation of (6) yields a majorant $V_{1}(z)$ defined throughout $\bar{R}$ and which may replace $V(z)$ in Theorem 1 . We require that $V_{1}(z)$ shall be harmonic in $R$, continuous in $\bar{R}$, equal to $\log M$ as defined by (7) on the component or components of $B$ on which $V(z)$ takes its maximum value in $\bar{R}$, and equal to $V(z)$ on the remaining components of $B$. In $R$ we have (12) as before. It follows from Theorem 1 that when $Q_{0}$ lies in $R$ the first member of (5) is not greater than $\left[\max e^{V_{1}(z)}, z\right.$ on $\left.Q_{0}\right]$, which is less than the second member of (5); this contradicts (5) and thereby establishes (6).

If $Q$ in $\bar{R}$ is not identical with $\bar{R}$ but intersects $B$, the set $Q$ separates $R$ into one or more subregions each containing no point of $Q$. Denote by $R_{1}$ an arbitrary one of these subregions. At least one boundary point of $R_{1}$ is a point of $Q$. Of course we suppose (7), (8), and (9) valid on $Q$ as before; we are to reach a contradiction.

We assume as before that the complement of $Q$ is simply connected, and assume also that $Q$ intersects but one component of $B$. Let $B_{1}$ be the boundary of $R_{1}$. We denote by $V_{1}(z)$ the function bounded and harmonic in $R_{1}$, continuous and equal to $\log M$ in the points of $B_{1}$ which belong to $Q$, and continuous and equal to $V(z)$ in the points of $B_{1}$ not points $A_{j}$ and not belonging to $Q$. Whether or not $B_{1}$ lies entirely in $B$, some component of $B_{1}$ contains some point $D$ of $B_{1}$ such that every point of $B_{1}$ in a neighborhood of $D$ belongs to $Q$. Map onto the interior of a circle $C$ the complement of that component of $B_{1}$ (there can be but one such component) which contains points of $Q$. In this conformal map the boundary elements (prime ends) of $R_{1}$ which consist of points of $Q$ correspond to precisely an entire arc of $C$, possibly the entire circumference. From this map it becomes clear that Theorem 1 in generalized 
form applies to the region $R_{1}$, and that (12) is valid in $R_{1}$. Consequently we have (compare (11))

$$
\limsup _{n \rightarrow \infty}\left[\max \left|F_{n}(z)\right|, z \text { on } Q_{1}\right] \leqq\left[\max e^{V_{1}(z)}, z \text { on } Q_{1}\right] \text {, }
$$

provided merely that $Q_{1}$ is an arbitrary continuum which lies in $R_{1}$. Then $Q_{1}$ also lies in $R$, and inequalities (12) and (13) contradict (6) as already established for a continuum which lies in $R$. Theorem 2 has now been completely proved.

It is to be noted that under the conditions of Theorem 2 the conclusion (6) may fail if we do not suppose $Q$ to consist of more than one point. This is illustrated by the example $R:|z|<1, F_{n}(z) \equiv z^{1 / n}, V(z) \equiv 0$. On every continuum $Q$ in $\bar{R}$ consisting of more than one point the first member of (6) has the value unity; but if $Q$ reduces to the single point $z=0$, the first member of (6) is zero.

We shall now apply Theorems 1 and 2 to a configuration in which the function $V(z)$ takes on only two distinct values on $B$, and in which the loci $V(z)=$ const. in $R$ have especially simple topological properties. This configuration occurs rather frequently in various studies on interpolation and approximation (loc. cit.).

THEOREM 3. Let $R$ be a region bounded by a finite number of mutually disjoint Jordan curves $K_{0}$ and a finite number of mutually disjoint Jordan curves $K_{-1}$ disjoint with $K_{0}$. Let the function $U(z)$ be harmonic in $R$, continuous in the corresponding closed region $\bar{R}$, equal to zero and -1 on $K_{0}$ and $K_{-1}$ respectively. Denote generically by $K_{\sigma}$ the locus $U(z)=\sigma, 0>\sigma>-1$, in $R$, by $R_{\sigma}$ the open set $\sigma>U(z)>-1$ in $R$ bounded by $K_{\sigma}$ and $K_{-1}$; then $R_{\sigma}$ is a sum of regions; the closure of $R_{\sigma}$ is denoted by $\bar{R}_{\sigma}$.

Let the function $f(z)$ be analytic throughout $R_{\rho}$ but (considered with its possible analytic extensions) not be analytic throughout any $R_{\rho^{\prime}}$ with $0>\rho^{\prime}>\rho$, and let $f(z)$ be continuous in the two-dimensional sense on $K_{-1}$ with respect to the domain $\bar{R}$. Let the functions $f_{n}(z)$ for $n=1,2, \cdots$ be analytic in $R$, continuous in $\bar{R}$, with

$$
\begin{array}{r}
\limsup _{n \rightarrow \infty}\left[\max \left|f_{n}(z)\right|, z \text { on } K_{0}\right]^{1 / n} \leqq e^{\alpha}>1, \\
\limsup _{n \rightarrow \infty}\left[\max \left|f(z)-f_{n}(z)\right|, z \text { on } K_{-1}\right]^{1 / n} \leqq e^{\beta}<1 .
\end{array}
$$

Then we must have

$$
\alpha+\alpha \rho-\beta \rho \geqq 0:
$$

moreover we have 


$$
\underset{n \rightarrow \infty}{\lim \sup }\left[\max \left|f_{n}(z)\right|, z \text { on } K_{\mu}\right]^{1 / n} \leqq e^{\alpha+\alpha \mu-\beta \mu},
$$

provided $\alpha+\alpha \mu-\beta \mu \geqq 0$ (this inequality is satisfied if we have $0 \geqq \mu \geqq \rho$ ), and we have

$$
\limsup _{n \rightarrow \infty}\left[\max \left|f(z)-f_{n}(z)\right|, z \text { on } K_{\sigma}\right]^{1 / n} \leqq e^{\alpha+\alpha \sigma-\beta \sigma},
$$

provided $\alpha+\alpha \sigma-\beta \sigma<0$ (this inequality is satisfied for $\sigma$ sufficiently near -1 ). If the equality sign holds in (16):

$$
\alpha+\alpha \rho-\beta \rho=0,
$$

then the equality sign holds also in (17) and (18):

$$
\begin{aligned}
& \underset{n \rightarrow \infty}{\lim \sup }\left[\max \left|f_{n}(z)\right|, z \text { on } K_{\mu}\right]^{1 / n}=e^{(\alpha-\beta)(\mu-\rho)}, \quad 0 \geqq \mu \geqq \rho, \\
& \limsup _{n \rightarrow \infty}\left[\max \left|f(z)-f_{n}(z)\right|, z \text { on } K_{\sigma}\right]^{1 / n}=e^{(\alpha-\beta)(\sigma-\rho)}, \quad \rho>\sigma \geqq-1 \text {. }
\end{aligned}
$$

Indeed, if $Q_{\sigma}$ is a continuum in $\bar{R}$ consisting of more than one point and lying in $\bar{R}_{\sigma}$ but having at least one point in common with $K_{\sigma}$, then we have

$$
\limsup _{n \rightarrow \infty}\left[\max \left|f_{n+1}(z)-f_{n}(z)\right|, z \text { on } Q_{\sigma}\right]^{1 / n}=e^{(\alpha-\beta)(\sigma-\rho)},
$$

and we have

$$
\begin{array}{ll}
\limsup _{n \rightarrow \infty}\left[\max \left|f(z)-f_{n}(z)\right|, z \text { on } Q_{\sigma}\right]^{1 / n}=e^{(\alpha-\beta)(\sigma-\rho)} & \text { if } \sigma<\rho, \\
\limsup _{n \rightarrow \infty}\left[\max \left|f_{n}(z)\right|, z \text { on } Q_{\sigma}\right]^{1 / n}=e^{(\alpha-\beta)(\sigma-\rho)} & \text { if } \sigma \geqq \rho .
\end{array}
$$

From the general properties of harmonic functions it follows (as in op. cit., $\$ \$ 3.3$ and 4.1) that each $K_{\sigma}$ in $R$ consists of a finite number of disjoint analytic Jordan curves, except that for each of a finite number of values of $\sigma$ the curves may have a finite number of multiple points. At such a multiple point the tangents to the various branches of $K_{\sigma}$ are equally spaced. Each $K_{\sigma}$ separates each point of $K_{0}$ from each point of $K_{-1}$. The open set $R_{\sigma}$ is not necessarily connected, but consists of one or more regions, each bounded by points of $K_{\sigma}$ and points of $K_{-1}$.

It is not the intention in Theorem 3 to demand that $f(z)$ shall be a monogènic analytic function. The function $f(z)$ shall be single-valued and analytic at every point of $R_{\rho}$, but there shall be no function single-valued and analytic throughout the interior of any $R_{\rho^{\prime}}, \rho^{\prime}>\rho$, which coincides with $f(z)$ in $R_{\rho}$. Thus (a) some branch of $f(z)$ found by analytic extension in $R_{\rho}$ from the neighborhood of $K_{-1}$ has a singularity on $K_{\rho}$; or (b) the locus $K_{\rho}$ in $R$ has a 
multiple point, and the monogenic functions defined at that multiple point by analytic extension in the various separated parts of $R_{\rho}$ from the neighborhood of $K_{-1}$ are not identical; or (c) both (a) and (b) occur.

Of course $\alpha+\alpha \sigma-\beta \sigma$ is merely a linear function of $\sigma$, which for $\sigma=0$ reduces to $\alpha$ and for $\sigma=-1$ reduces to $\beta$.

From inequality (14) we may write for arbitrary $\epsilon>0$ and for $n$ sufficiently large

$$
\begin{array}{cc}
\left|f_{n}(z)\right| \leqq e^{n(\alpha+\epsilon)}, \quad\left|f_{n+1}(z)\right| \leqq e^{n(\alpha+\epsilon)}, & z \text { on } K_{0}, \\
\left|f_{n+1}(z)-f_{n}(z)\right| \leqq 2 e^{n(\alpha+\epsilon)}, & z \text { on } K_{0}, \\
\limsup \left[\max \left|f_{n+1}(z)-f_{n}(z)\right|, z \text { on } K_{0}\right]^{1 / n} \leqq e^{\alpha} . &
\end{array}
$$

From inequality (15) we may similarly write

$$
\underset{n \rightarrow \infty}{\lim \sup }\left[\max \left|f_{n+1}(z)-f_{n}(z)\right|, z \text { on } K_{-1}\right]^{1 / n} \leqq e^{\beta} .
$$

We are now in a position to apply Theorems 1 and 2, identifying the region $R$ of Theorem 1 with the region $R$ of Theorem 3, setting

$$
F_{n}(z) \equiv\left[f_{n+1}(z)-f_{n}(z)\right]^{1 / n}
$$

and setting

$$
V(z) \equiv \alpha+(\alpha-\beta) U(z) .
$$

Inequality (2) yields at once $(0>\sigma>-1)$

$$
\limsup _{n \rightarrow \infty}\left[\max \left|f_{n+1}(z)-f_{n}(z)\right|, z \text { on } K_{\sigma}\right]^{1 / n} \leqq e^{\alpha+\alpha \sigma-\beta \sigma} \text {. }
$$

We are now in a position to establish (16). It follows from (15) that $f_{n}(z)$ approaches $f(z)$ uniformly on $K_{-1}$. If (16) is not true, we have $\alpha+\alpha \rho-\beta \rho<0$, from which it follows that we have $\alpha+\alpha \rho_{1}-\beta \rho_{1}<0$ for a suitably chosen $\rho_{1}$, $0>\rho_{1}>\rho$. Then by (24) for $\sigma=\rho_{1}$, the sequence $f_{n}(z)$ converges uniformly on $K_{\rho_{1}}$, hence converges uniformly in the closed set $\bar{R}_{\rho_{1}}$, to some function which we denote by $F(z)$, analytic in $R_{\rho_{1}}$ and continuous in $\bar{R}_{\rho_{1}}$. The function $F(z)-f(z)$ is analytic in $R_{\rho}$, continuous on $K_{-1}$ with respect to the region $R$, and vanishes on $K_{-1}$. Consequently (see for instance op. cit., §1.9) the function $F(z)-f(z)$ vanishes identically in the neighborhood of $K_{-1}$ interior to $R$, and vanishes identically whenever defined by analytic extension from that neighborhood. The function $F(z)$ is single-valued and analytic throughout

* It may seem to the reader somewhat clumsy to employ the values zero and -1 for $U(z)$ on the two parts of the boundary of $R$. Such choice is, however, rather convenient in the applications of Theorem 3. If any other two constants are used instead, conditions (16) and (16') are easily transformed into the new notation by means of the linearity property. 
$R_{\rho_{1}}$; hence $f(z)$ can be defined so as to be single-valued and analytic throughout $R_{\rho_{1}}$, contrary to hypothesis. This contradiction completes the proof of (16).

Inequality (24) implies for arbitrary $\epsilon>0$ and for $n$ sufficiently large

$$
\left|f_{n+1}(z)-f_{n}(z)\right| \leqq e^{(\alpha+\alpha \sigma-\beta \sigma+\epsilon) n}, \quad z \text { on } K_{\sigma},
$$

so merely by writing

$$
\begin{aligned}
f_{n+1}(z) \equiv f_{N}(z)+\left[f_{N+1}(z)-f_{N}(z)\right] & +\left[f_{N+2}(z)-f_{N+1}(z)\right] \\
+ & \cdots+\left[f_{n+1}(z)-f_{n}(z)\right], \quad n \geqq N,
\end{aligned}
$$

we deduce from (25) inequality (17), for all values of $\mu$ for which $\alpha+\alpha \mu-\beta \mu$ is nonnegative, hence certainly for all $\mu$ satisfying the inequality $0 \geqq \mu \geqq \rho$.

If we have $\alpha+\alpha \sigma-\beta \sigma<0$, and if $\epsilon>0$ is chosen so small that also $\alpha+\alpha \sigma-\beta \sigma+\epsilon<0$, then by (25) the sequence $f_{n}(z)$ converges uniformly on $K_{\sigma}$, hence by (15) converges uniformly in $\bar{R}_{\sigma}$ to some function $F(z)$ which by the reasoning used above is identical with $f(z)$ in $\bar{R}_{\sigma}$. Inequality (25) holds for $n$ sufficiently large, and the identity

$$
f(z)-f_{n}(z) \equiv\left[f_{n+1}(z)-f_{n}(z)\right\rfloor+\left\lfloor f_{n+2}(z)-f_{n+1}(z)\right]+\cdots
$$

then gives us inequality (18).

If the equality sign holds in (16), that is to say if $\left(16^{\prime}\right)$ is valid, we proceed to establish

$$
\limsup _{n \rightarrow \infty}\left[\max \left|f_{n+1}(z)-f_{n}(z)\right|, z \text { on } K_{\rho}\right]^{1 / n}=1 .
$$

If (28) does not hold, it follows from (25) that we have

$$
\limsup _{n \rightarrow \infty}\left[\max \left|f_{n+1}(z)-f_{n}(z)\right|, z \text { on } K_{p}\right]^{1 / n} \leqq e^{\gamma}<1 ;
$$

we show that (29) leads to a contradiction. Apply Theorem 1 to the region or regions in $R$ between and bounded by $K_{0}$ and $K_{\rho}$, setting as before

$$
F_{n}(z) \equiv\left[f_{n+1}(z)-f_{n}(z)\right]^{1 / n},
$$

and now with the choice

$$
V(z) \equiv \alpha+(\gamma-\alpha) U(z) / \rho .
$$

By virtue of (22) and (29), Theorem 1 applies here. On $K_{\rho}$ we have $V(z)=\gamma<0$, so on a suitably chosen $K_{\rho_{1}}$ with $0>\rho_{1}>\rho$ we also have $V(z)<0$. The corresponding inequality (2) for each component of $K_{\rho_{1}}$ implies uniform 
convergence of the sequence $f_{n}(z)$ on $K_{\rho_{1}}$, which we have already shown to be impossible. This contradiction establishes equation (28).

Theorem 2 now applies to the original region $R$, with $V(z) \equiv \alpha+(\alpha-\beta) U(z)$, thanks to (28) as a consequence of $\left(16^{\prime}\right)$, and establishes from (22) and (23) the equation

(30) $\quad \limsup \left[\max \left|f_{n+1}(z)-f_{n}(z)\right|, z \text { on } K_{\sigma}\right]^{1 / n}=e^{(\alpha-\beta)(\sigma-\rho)}, \quad 0 \geqq \sigma \geqq-1$.

By use of (26) and (27) respectively, equation (30) implies

$$
\begin{array}{rr}
\limsup _{n \rightarrow \infty}\left[\max \left|f_{n}(z)\right|, z \text { on } K_{\mu}\right]^{1 / n} \leqq e^{(\alpha-\beta)(\mu-\rho)}, & 0 \geqq \mu \geqq \rho, \\
\lim \sup \left[\max \left|f(z)-f_{n}(z)\right|, z \text { on } K_{\sigma}\right]^{1 / n} \leqq e^{(\alpha-\beta)(\sigma-\rho)}, & \rho>\sigma \geqq-1 .
\end{array}
$$

On the other hand, elementary inequalities show that the inequality sign in (31) or (32) would contradict (30). Consequently equations $\left(17^{\prime}\right)$ and (18') are established.

Equation (19) follows from equation (28) and Theorem 2 applied to the original region $R$ by use of (22) and (23). Equations (20) and (21) follow from (19) precisely as we have derived $\left(17^{\prime}\right)$ and $\left(18^{\prime}\right)$ from (30). Theorem 3 is completely proved.

In connection with Theorem 3 it may be noticed that inequalities (17) and (18) may both be expressed in essence in the form

$$
\underset{n \rightarrow \infty}{\limsup }\left[\max \left|f_{n+1}(z)-f_{n}(z)\right|, z \text { on } K_{\sigma}\right]^{1 / n} \leqq e^{\alpha+\alpha \sigma-\beta \sigma},
$$

with no restriction that $\alpha+\alpha \sigma-\beta \sigma$ should be positive, negative, or zero. But (18) is more specific than (33), in the sense that (18) exhibits the limit $f(z)$ of the sequence $f_{n}(z)$ if the second member of (18) is less than unity. Similarly, inequalities $\left(17^{\prime}\right)$ and $\left(18^{\prime}\right)$ may be combined in the single form (30), which is true for every $\sigma, 0 \geqq \sigma \geqq-1$, provided $\left(16^{\prime}\right)$ is valid. We formulate

COROLlARY 1. Under the hypothesis of Theorem 3, inequality (33) is valid for every $\sigma, 0 \geqq \sigma \geqq-1$; if equation (16') holds, then equation (30) is valid for every $\sigma, 0 \geqq \sigma \geqq-1$.

In the nature of a converse to Corollary 1 we have

CoRollary 2. Let $R$ and $f(z)$ satisfy the hypothesis of Theorem 3. Let the sequence of functions $f_{n}(z)$ analytic in $R$ and continuous in $\bar{R}$ converge uniformly to $f(z)$ on $K_{-1}$, and satisfy the inequalities (22) and (23), with $\alpha>0, \beta<0$. Then the conclusions of Theorem 3 and of Corollary 1 are fulfilled.

The proof of Corollary 2 is contained in our previous discussion, for we 
have used essentially (22) and (23) rather than (14) and (15) in the proof of Theorem 3.

For the sake of simplicity we have assumed in Theorem 3 and its corollaries that $R$ is bounded by a finite number of mutually disjoint Jordan curves $K_{0}$ and a finite number of mutually disjoint Jordan curves $K_{-1}$ disjoint with $K_{0}$; and have assumed $f(z)$ to be continuous on $K_{-1}$ in the two-dimensional sense with respect to $\bar{R}$, and $f_{n}(z)$ to be continuous in $\bar{R}$. Theorem 3 and its corollaries persist if these requirements are somewhat weakened, namely replaced by the requirement that $R$ be bounded by two disjoint sets $K_{0}$ and $K_{-1}$, each of which consists of a finite number of components none of which is a single point; and the requirement that $f(z)$ is bounded in the neighborhood of $K_{-1}$ in $R$, and that $f_{n}(z)$ is bounded in $R$. Under these new conditions on $R$ it is no loss of generality to assume that $R$ fulfills the original conditions, for the method of successive conformal mapping onto the interior of a circle of simply-connected regions containing $R$ each bounded by a single component of the boundary of $R$ maps $R$ onto a region fulfilling the original requirements on $R$ and which is even bounded by a finite number of analytic Jordan curves. The function $U(z)$ is invariant under such transformation. In such expressions as appear in (14), (15), (22), (23), the term " $\max$ " is now to be interpreted as the least upper bound of the limit values as $z$ in $R$ approaches $K_{0}$ or $K_{-1}$. We may also take here not least upper bound of all limit values, but least upper bound of boundary values for normal approach in the sense of Fatou; these values exist almost everywhere (we assume $R$ bounded by analytic Jordan curves), and may be used also in Theorem 1; the least upper bound of limit values cannot exceed the least upper bound of Fatou boundary values. With the new hypothesis and this new interpretation of notation, the previous reasoning remains essentially valid.*

It is fairly obvious that Theorem 3 extends to the case that $K_{0}$ and $K_{-1}$ may have a finite number of points in common. But here the topological nature of the loci $K_{\sigma}$ may become rather complicated. Indeed, Theorem 3 extends also to the case that $U(z)$ is no longer piecewise constant on the boundary $B$ of $R$, but is bounded in $R$ and continuous in $\bar{R}$ except at a finite number of points. Under these conditions we replace inequalities (22) and (23) of Corollary 2 by such a relation as

* We need, however, some such proposition as the following: If the function $\phi(z)$ is analytic and bounded for $r \leqq|z|<1$, and if the boundary values of $\phi(z)$ for normal approach to $|z|=1$ vanish almost everywhere on $|z|=1$, then $\phi(z)$ vanishes identically. Form Cauchy's integral for $z$ in the annular region $r \leqq|z| \leqq r_{1}<1$, the sum of an integral over $|z|=r$ and an integral over $|z|=r_{1}$; as $r_{1}$ approaches unity the Cauchy integral for $\phi(z)$ over $|z|=r_{1}$ approaches zero and therefore vanishes identically; hence $\phi(z)$ can be extended analytically across $|z|=1$ so as to be analytic for every $z$ with $|z| \geqq r$. Since $\phi(z)$ has the value zero almost everywhere on the circle $|z|=1$, that function vanishes identically. 


$$
\underset{n \rightarrow \infty}{\lim \sup }\left\{\text { l.u.b. }\left[e^{U(z)}-\left|f_{n+1}(z)-f_{n}(z)\right|^{1 / n}\right], z \text { on } B\right\} \geqq 0 \text {, }
$$

where as before the square bracket refers to limiting values on $B$. Theorems 1 and 2 admit a corresponding extension, where $V(z)$ is merely harmonic and bounded in $R$, and continuous in $\bar{R}$ except at a finite number of points.

We establish still another corollary to Theorem 3:

Corollary 3. Under the conditions of Theorem 3 with (16') fulfilled, the sequence $f_{n}(z)$ converges throughout no region lying in $R$ but not in $R_{\rho}$.

If the sequence $f_{n}(z)$ converges in a region which lies in $R$ but not in $R_{\rho}$, that sequence converges uniformly in a region $Q$ which lies in $R$ but not in $R_{\rho}$, for it follows from a theorem due to Osgood that every region of convergence contains a region of uniform convergence. For the particular region $Q$ we have

$$
\underset{n \rightarrow \infty}{\limsup }\left[\max \left|f_{n+1}(z)-f_{n}(z)\right|, z \text { on } Q\right]^{1 / n} \leqq 1,
$$

in contradiction to (19).

The two-constant theorem can be employed with ease in the proof of Theorem 3. Indeed, the conclusions (16), (17), (18), (17'), (18') can be established by the method used for the preliminary results (in the papers already mentioned) which suggested Theorem 3 and are contained in it. Also the conclusions (19), (20), (21) can be proved by the two-constant theorem provided $\sigma>-1$, by an extension of that same method. These proofs are less immediate than the present ones, namely applications of Theorem 2. The writer doubts that (19), (20), (21) can be proved by the two-constant theorem if $\sigma=-1$, that is to say, if $Q_{\sigma}$ is an $\operatorname{arc}$ of $K_{-1}$.

HARVARD UNIVERSITY, Cambridge, Mass. 